\title{
TACHIRAMANTIS DOUGLASI (ANURA: CRAUGASTORIDAE)
}

Diego Amaya-Villabona ${ }^{1}$ Y JAVIER E. Cortes-SuÁREZ ${ }^{2 *}$

${ }^{1}$ Laboratorio de Ecología y Modelación del Paisaje, Universidad Nacional de Colombia, Sede Bogotá, Bogotá D.C, Colombia.

${ }^{2}$ Fundación Ecohumana para la Promoción del Desarrollo Humano Integral Sostenible. Villa de Leyva, Boyacá, Colombia.

"Correspondent:javi1885@gmail.com

Colombia: Santander: Municipio de Charta, Finca Arizona (7.293880 N; -72.957770 O, WGS 84, $2331 \mathrm{msnm}$ ), 24 de julio de 2018 entre las 19:00-21:00 h. Este reporte representa el primer registro de la especie en el municipio de Charta en Santander. Se encuentra localizado a $9.5 \mathrm{Km}$ de distancia de la localidad conocida más cercana donde se ha registrado previamente la especie en el municipio de Suratá en Santander dirección Noreste (GBIF, 2018; SiBColombia, 2018). Se registraron a través de vocalizaciones y observación directa dos machos adultos de Tachiramantis douglasi a nivel del estrato arbustivo sobre la vegetación entre un borde de bosque y una matriz de pastizal utilizada para ganadería ocasional (Fig. 1). El registro de T. douglasi en el municipio de Charta amplía el rango de distribución conocido para la especie (Fig. 2), teniendo en cuenta que sólo había sido reportada para el departamento de Santander en los municipios de Piedecuesta (Arroyo et al., 2008, 2013; GBIF, 2018; SiBColombia, 2018), Floridablanca, Tona y Suratá (GBIF, 2018; SiBColombia, 2018). Igualmente, el presente registro amplia el área de distribución de la especie fuera de áreas protegidas



Figure 1. Individual of T. douglasi and panoramic view of the habitat where it was registered in the Arizona farm, municipality of Charta, Santander. A) Adult male found on shrub vegetation (Reference No. 14863089, iNaturalist photo catalog). B) Edge of forest and grassland matrix. Photo: Diego Amaya-Villabona.

Figura 1. Individuo de T. douglasi y panorámica del hábitat donde fue registrado en la Finca Arizona, municipio de Charta, Santander. A) Macho adulto encontrado sobre la vegetación arbustiva (No de referencia 14863089, catálogo de fotografías de iNaturalist). B) Borde de bosque y matriz de pastizal. Foto: Diego Amaya-Villabona.

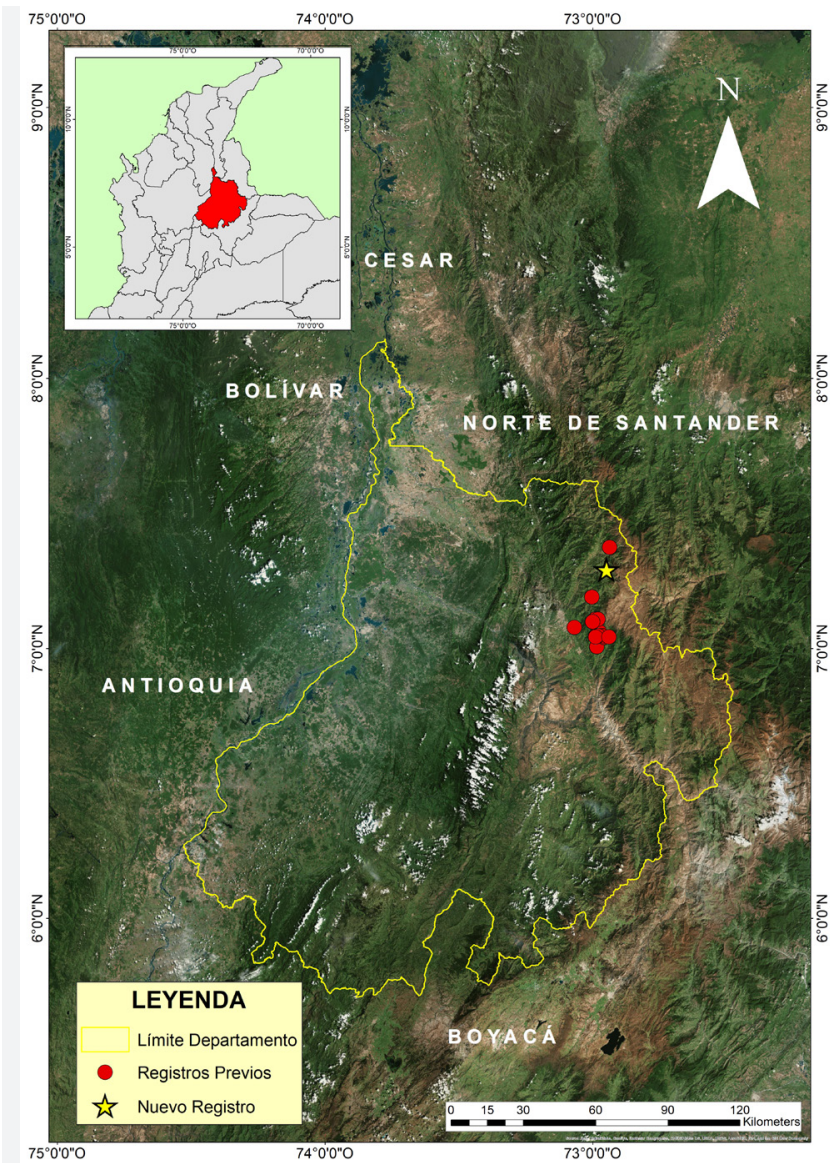

Figure 2. Geographical distribution known in Santander (Arroyo et al., 2008, 2013; GBIF, 2018; SiBColombia, 2018), and new record of T. douglasi in the municipality of Charta.

Figura 2. Distribución geográfica conocida en Santander (Arroyo et al., 2008, 2013; GBIF 2018; SiBColombia, 2018), y nuevo registro de T. douglasi en el municipio de Charta.

donde ha sido registrada en el departamento, tales como, los Parques Naturales Regionales: Bosques Andinos Húmedos el Rasgón (Estación experimental y demostrativa "El Rasgón") en el municipio de Piedecuesta (Arroyo et al., 2008, 2013; AlbornozEspinel et al., 2017), Cerro de la Judía en Floridablanca y Piedecuesta, y Santurbán en los municipios de Suratá, California y Vetas (Albornoz-Espinel et al., 2017; Grupo Áreas Protegidas 
Corponor, 2018), los cuales se encuentran asociados al Complejo de Páramos Jurisdicciones Santurbán-Berlín. Los especímenes fueron fotografiados por Diego Amaya-Villabona y verificados por Andrés Mauricio Forero y Javier Cortés-Suárez. El registro fotográfico del espécimen en esta nota se encuentra depositado con número de referencia 14863089 en el catálogo de fotografías de "iNaturalist", iniciativa de la California Academy of Sciences y la National Geographic Society.

\section{LITERATURA CITADA}

Albornoz-Espinel, M.M., C. Cáceres \& A.A. Acevedo. 2017. Protected areas assessment for the conservation of threatened amphibians in the Cordillera Oriental of Colombia. Herpetology Notes 10:685-696.

Arroyo, S., A. Jerez \& M.P. Ramírez-Pinilla. 2013. Anuros de un bosque de niebla de la Cordillera Oriental de Colombia. Caldasia 25(1): 153-167.
Arroyo, S. B., V. H. Serrano-Cardozo \& M.P. Ramírez-Pinilla. 2008. Diet, microhabitat and time of activity in a Pristimantis (Anura, Strabomantidae) assemblage. Phyllomedusa 7(2):109-119.

GBIF. 2018. Pristimantis douglasi occurrence data. https://doi. org/10.15468/dl.4p7mdk [Accessed on September 2018]

GrupoÁreas Protegidas Corponor. 2018. Sistema de Paramos delGran Santander. http://corponor.gov.co/areasnaturalesestrategicas/ descargas/mapa_Santurban_Almorzadero_general.pdf [Consultado en Septiembre 2018]

SiBColombia. 2018. Sistema de Información sobre Biodiversidad de Colombia. http://www.sibcolombia.net/web/sib/cifras [Consultado en Abril 2018]. 